\title{
Spectral Geometry of Black Holes in $4 D$ Gauged Supergravity
}

\author{
P. Bargueño ${ }^{1, *}$, E. Contreras ${ }^{2}$ and J.M. Peña ${ }^{3}$ \\ 1 Departamento de Física Aplicada, Campus de San Vicente del Raspeig, Universidad de Alicante, \\ E-03690 Alicante, Spain \\ 2 Departamento de Física, Colegio de Ciencias e Ingeniería, Universidad San Francisco de Quito, \\ Quito 170901, Ecuador; econtreras@usfq.edu.ec \\ 3 Departamento de Física, Universidad de Antofagasta, Aptdo 02800, Chile; joselen@yahoo.com \\ * Correspondence: pedro.bargueno@ua.es
}

check for

updates

Citation: Bargueño, P.; Contreras, E.; Peña, J.M. Spectral Geometry of Black Holes in $4 D$ Gauged Supergravity. Universe 2021, 7, 78. https://doi.org/ $10.3390 /$ universe7040078

Academic Editor: Lorenzo Iorio

Received: 2 February 2021

Accepted: 21 March 2021

Published: 25 March 2021

Publisher's Note: MDPI stays neutral with regard to jurisdictional claims in published maps and institutional affiliations.

Copyright: (C) 2021 by the authors. Licensee MDPI, Basel, Switzerland. This article is an open access article distributed under the terms and conditions of the Creative Commons Attribution (CC BY) license (https:/ / creativecommons.org/licenses/by/ $4.0 /)$.

\begin{abstract}
In this work we show that the area of the event horizon of the Chong-Cvetic-Lu-Pope black hole can be reconstructed in terms of the spectra of the Laplacian on the event horizon. This result, which extends previous works regarding the de Sitter-Kerr-Newman case, points towards a possible description of the black hole degrees of freedom in terms of the spectra of canonical operators defined on the event horizon.
\end{abstract}

Keywords: black holes; supergravity; spectral geometry

\section{Introduction}

The long-sought unification of General Relativity (GR) and Quantum Theory (QT) into a full theory of Quantum Gravity (QG) remains, up today, elusive. If the language of both GR and QT should be linked at some level in order to incorporate the essentials of both theories, Spectral Geometry (SG) emerges as the natural arena to formulate QG: geometry coming from GR and functional analysis coming from QT. Roughly speaking, SG tries to describe the shape of a manifold through its vibrational spectrum. Although the subject was firstly introduce in pure mathematics by Weyl [1] (see, for example, [2]), it started to bring the attention of the physics community working in gravitation in the late 90s from the works of Chamseddine and Connes [3,4] and Rovelli [5]. Recently, it has been pushed forward by Kempf and coworkers [6,7]. For QG, SG tries to answer to what extent the curvature of compact $n$-dimensional Riemannian manifolds without boundaries can be described in terms of the spectra of canonical differential operators (Laplace or Dirac, for example) defined on such a manifold. Although the Lorentzian case has been studied to a lesser extent [8] than the Riemannian version, the later remains of interest within the Euclidean approach to QG. In addition, the degrees of freedom of gravity would be cleanly identified as the relevant set of eigenvalues which, being invariants, would be then free from important problems such as non-diffeomorphism invariance, for example. However, although SG offers a promising new look at QG, the question of the existence of isospectral but non-isometric manifolds [9], together with the almost intractabilility of the problem in the generic case of manifolds without symmetries representing a general space-time, makes the approach difficult to implement, although several advances has been recently achieved [10].

Regarding black holes (BHs), whose role as keepers of the secrets of QG are free from any doubt, SG has been scarcely used to disentangle their properties. In particular, Engmann and coworkers [11] explicitly reconstructed the metric of the Kerr-Newman event horizon from the spectrum of its Laplacian. Even more, they gave an unique and explicit determination of the metric for the uncharged case from the aforementioned spectrum, which they interpreted, by using Robinson's uniqueness theorem [12], as if "One can hear the shape of noncharged stationary axially symmetric $\mathrm{BH}$ space-times by listening to the vibrational frequencies of its event horizon only" [11]. This result was later extended to 
the de Sitter-Kerr-Newman case [13], showing that the metric is uniquely determined by the union of the spectra of its cosmological and event horizons. Interestingly, the authors concluded in Ref. [11] by noting that their main results are consistent with the holographic principle since the whole metric space-time structure can be encoded in the intrinsic spectral data of the corresponding two-dimensional event horizon surface, suggesting that the spectrum of the Laplacian on event horizons should playing an important and unnoticed role in the space-time manifold.

The purpose of this work is twofold: (i) to extend Engmann's works to more complex $\mathrm{BH}$ solutions beyond GR such as the Chong-Cvetic-Lu-Pope (CCLP) BH solution of $4 D$ gauged supergravity $[14,15]$ and (ii) to encourage the study of the role of the spectrum of the Laplacian on event horizons as possible BH degrees of freedom. The idea behind considering the CCLP solution is that, although it corresponds to a effective $4 D$ model, this is connected with theories which microscopical degrees of freedom are well-known (in principle). So, the use of SG in this context should give more confidence in to use it as a tool to explore how the quantum effects emerge in the underlying geometry. Besides, we are interested in to prove that we are able to express the horizon metric of theories beyond GR in an adequate form that could allow to identify the horizon area in a straightforward way as in [11], for example.

This work is organized as follows. Section 2 summarizes the main features of supergravity theories having the (CCLP) as a particular solution, which is later described using some convenient variables in Section 3. The main calculation of the spectra of the event horizon is developed in Section 4, while the discussion and conclusions are left to Section 5.

\section{Gauged and Ungauged Supergravities in $4 D$}

The supergravity theories are theories with local supersymmetry [16]. They are effective background theories which have correspondence with strings $(D=10), M 2$ branes and p-branes (in $D=11$ and other dimensions, respectively), see for example [17]. The 11dimensional supergravity is unique and corresponds to the low energy effective theory of the conjectured M-theory, which should be a theory of everything [18]. Supergravities can be formulated in various space-time dimensions and they can be ungauged or gauged (in the literature we can also find the massive supergravities that are deformations of an ungauged supergravity where some fields acquire mass), and depending on supersymmetries allowed, they can be called as maximal, half-maximal and so on.

In the ungauged supergravities, the matter fields are not charged under the abelian gauge group, while the gauged ones come with non-abelian groups under which the matter fields are charged, see [19]. The gauged supergravities are deformations of ungauged ones. There are different ways to obtain ungauged and/or gauged supergravities in diverse dimensions $D<11$. In the case of ungauged supergravities, it can start from a $D=11$ maximal supergravity and reducing dimensionally on a torus $T^{n}$ we obtain ungauged supergravities in $(D-n)$ dimensions. On the other hand, the type Scherk-Schwarz dimensional reductions, the compactifications using geometric and non-geometric fluxes, and the compactifications in presence of $\mathrm{p}$-forms fluxes, produce gauged supergravities, from ungauged ones, in lower dimensions [19]. For a complete review on supergravities and their solutions in $D=4$ see [20].

The $\mathrm{BH}$ solution appears in supergravity theories in diverse dimensions [20]. There are different ways to construct these solutions. In the string context there exists the socalled solution-generating technique (for a easy description see [21]), that generates a new solution (from a known $\mathrm{BH}$ one) which in general has charges associated with it.

In this work, we use a BH solution constructed using the method in $[14,15]$ for $N=4$, $D=4$ gauged supergravity. It is a charged rotating $\mathrm{BH}$, with four charges pairwise equal. This solution verifies the equations of motion that are coming from a lagrangian which is a consistent bosonic truncation of $S O(4) N=4$ gauged supergravity, where only the $U(1) \times U(1)$ charged abelian fields are included. In order to construct it, the authors 
considered the form of Kerr-Newman-AdS BH and the solution with four charges pairwise equal from $N=4, D=4$ ungauged supergravity [14,15].

Specifically, the $N=4 D=4$ bosonic CCLP Lagrangian is

$$
\begin{aligned}
L=R * 1-\frac{1}{2} * d \varphi_{1} & \wedge d \varphi_{1}-\frac{1}{2} e^{2 \varphi_{1}} * d \chi_{1} \wedge d \chi_{1}-\frac{1}{2} e^{-\varphi_{1}}\left(* F_{(2) 1} \wedge F_{(2) 2}+* F_{(2) 2} \wedge F_{(2) 2}\right)+ \\
-\frac{1}{2} \chi_{1}\left(F_{(2) 1}\right. & \left.\wedge F_{(2) 1}+F_{(2) 2} \wedge F_{(2) 2}\right)-g^{2}\left(4+2 \cosh \varphi_{1}+e^{\varphi_{1}} \chi_{1}^{2}\right) * 1,
\end{aligned}
$$

where $\varphi_{1}, \chi_{1}, F_{(2) 1}=d A_{(1) 1}, F_{(2) 2}=d A_{(1) 2}$ are the dilaton, the axion and the field strengths, respectively. $g$ is a constant in the scalar potential.

The authors of Ref. [14] mention that this CCLP solution, when uplifted to $D=11$, represents a rotating M2-brane with rotations in the world-volume and the transverse space. They used the reduction ansatz applied by [22] to obtain $N=4$ gauged supergravity from $S^{7}$ reduction of $D=11$ supergravity. This is an interesting result because M2-brane plays a main role in M-Theory. Moreover, Stelle and Duff $[17,23]$ found a solution of $D=11$ supergravity that couples to a 2-brane charged as a source. Besides, this gauged supergravity solution provides new gravitational backgrounds for a further study of the $A d S_{4} / C F T_{3}$ given that such non-extremal solutions play an important role in the study of the dual field theory at non-zero temperature.

\section{The Chong-Cvetic-Lu-Pope Metric and Its Event Horizon}

The non-extremal rotating charged CCLP BH solutions we are interested in are given, in a frame that rotates at infinity, by $[14,15]$

$$
\begin{aligned}
d s^{2} & =-\frac{\Delta_{r}}{W}\left(d t-a \sin ^{2} \theta \Xi^{-1} d \phi^{2}\right)^{2}+W\left(\frac{d r^{2}}{\Delta_{r}}+\frac{d \theta^{2}}{\Delta_{\theta}}\right) \\
& +\frac{\Delta_{\theta} \sin ^{2} \theta}{W}\left[a d t-\left(r_{1} r_{2}+a^{2}\right) \Xi^{-1} d \phi^{2}\right],
\end{aligned}
$$

where

$$
\begin{aligned}
r_{i} & =r+2 m s_{i}^{2} \\
\Delta_{r} & \equiv r^{2}+a^{2}-2 m r+g^{2} r_{1} r_{2}\left(r_{1} r_{2}+a^{2}\right) \\
\Delta_{\theta} & =1-g^{2} a^{2} \cos ^{2} \theta \\
W & =r_{1} r_{2}+a^{2} \cos ^{2} \theta \\
\Xi & =1-a^{2} g^{2} .
\end{aligned}
$$

As usual, $m$ and $a$ are the mass and angular momentum parameter of the hole, while $s_{i}=\sinh \delta_{i}, c_{i}=\cosh \delta_{i}$, with $\delta_{i}$ being related to the charges of the solution. Finally, $g$ is related to the intensity of the scalar potential. As we are only interested in the gravitational sector of the solution, the corresponding solutions for the gauge, axion and dilaton fields will not be revised nor commented (the interested reader can find them in [14]).

Following Smarr [24], we define the scale parameter by

$$
\eta=\sqrt{r_{1}^{+} r_{2}^{+}+a^{2}}
$$

and the distortion parameter by

$$
\beta=\frac{a}{\sqrt{r_{1}^{+} r_{2}^{+}+a^{2}}},
$$

where $r_{i}^{+}=r^{+}+2 m s_{i}^{2}$ with $r^{+}$the horizon radius. Even more, we introduce a new parameter given by

$$
\xi=-\frac{a^{2} g^{2}}{1-a^{2} g^{2}}
$$


After defining $x=-\cos \theta$, the metric of the event horizon $(\mathrm{H})$, which is obtained by setting $d r=d t=0$, with $r=r^{+}$in Equation (1), can be written as

$$
d s_{H}^{2}=\eta^{2}(1-\xi)\left(\frac{d x^{2}}{f(x)}+f(x) d \phi^{2}\right),
$$

with

$$
f(x)=\frac{1-\xi\left(1-x^{2}\right)}{1-\beta^{2}\left(1-x^{2}\right)}\left(1-x^{2}\right)
$$

with $x \in(-1,1)$. Note that $f(-1)=0=f(1)$ and $f^{\prime}(-1)=2=-f^{\prime}(1)$. Realising that the metric in the event horizon can be written as in Equation (6) is the main technical contribution of this work.

With this writing at hand, the area of the event horizon, $\mathcal{A}_{\mathcal{H}}=\int_{-1}^{1} \int_{0}^{2 \pi} \sqrt{{ }^{(2)} g} d x d \phi$, where ${ }^{(2)} g$ stands for the determinant of the 2-metric, can be expressed as

$$
\mathcal{A}_{\mathrm{H}}=4 \pi \eta^{2}(1-\xi)
$$

\section{The Spectral Determination for the Event Horizon}

In this section, we review the techniques of Engman $[11,25,26]$ in order to apply them to the event horizon of the metric given by Equation (1).

Let us study the Laplacian

$$
\Delta_{H}=-\frac{1}{\sqrt{g}} \frac{\partial}{\partial x^{i}}\left(\sqrt{g} g^{i j} \frac{\partial}{\partial x^{j}}\right)
$$

on the event horizon.

In $(x, \phi)$ coordinates, we have

$$
\Delta_{H}=-\frac{\partial}{\partial x}\left(f(x) \frac{\partial}{\partial x}\right)-\frac{1}{f(x)} \frac{\partial^{2}}{\partial \phi^{2}} .
$$

We can separate variables to obtain that the eigenspace of $\Delta_{H}, E_{\lambda_{m}}$, is given by [27]

$$
E_{\lambda_{m}}=\bigoplus_{k=-m}^{k=m} e^{i k \phi} W_{k},
$$

where $W_{k}$ is the eigenspace of the operator

$$
L_{k}=-\frac{d}{d x}\left(f(x) \frac{d}{d x}\right)+\frac{k}{f(x)} .
$$

Note that the spectrum of the event horizon $(H), \operatorname{Spec}(H)$, is given by

$$
\operatorname{Spec}(H)=\bigcup_{k \in \mathbb{Z}} \operatorname{Spec}\left(L_{k}\right),
$$

with $L_{k}$ the spectrum of the ordinary differential operator given by

$$
\operatorname{Spec}\left(L_{k}\right)=\left\{0<\lambda_{k}^{1}<\lambda_{k}^{2}<\cdots<\lambda_{k}^{j}<\cdots\right\}
$$

for all $k \in \mathbb{Z}$.

Each $L_{k}$ has a Green operator, $\Gamma_{k}$, whose spectrum is [11]

$$
\operatorname{Spec}\left(\Gamma_{k}\right)=\left\{\frac{1}{\lambda_{k}^{j}}\right\}_{j=1}^{\infty} .
$$


Even more, the trace of $\Gamma_{k}$ is given by

$$
\gamma_{k}=\sum_{j=1}^{\infty} \frac{1}{\lambda_{k}^{j}}
$$

Finally, in Refs. [25,26] it is showed that, for a surface with a metric given by

$$
d s^{2}=\frac{d x^{2}}{f(x)}+f(x) d \phi^{2}
$$

we have

$$
\gamma_{0}=\frac{1}{2} \int_{-1}^{1} \frac{1-x^{2}}{f(x)} d x
$$

and

$$
\gamma_{k}=\frac{1}{|k|}, k \neq 0
$$

Then, as the metric of the event horizon and that of Equation (17) are related by

$$
d s_{H}^{2}=\eta^{2}(1-\xi) d s^{2},
$$

their spectra are related by

$$
\lambda \in \operatorname{Spec}\left(d s^{2}\right) \Longleftrightarrow \frac{\lambda}{\eta^{2}(1-\xi)} \in \operatorname{Spec}(H) .
$$

Therefore, using these techniques we get that, for the event horizon of the $\mathrm{BH}$ under consideration,

$$
\gamma_{0}=\eta^{2}\left[1-\beta^{2}+\left(\xi-\beta^{2}\right) g(\xi)\right]
$$

and

$$
\gamma_{k}=\frac{\eta^{2}(1-\xi)}{|k|}(k \neq 0)
$$

where we have defined an auxiliary function given by $g(\xi)=\frac{\left(\sqrt{\frac{1-\xi}{\xi}} \arctan \sqrt{\frac{\xi}{1-\xi}}\right)-1}{\xi}$.

Based on Equations (22)-(24), it can be shown that the area of the event horizon can be represented, for each $k \in \mathbb{N}$ as

$$
\mathcal{A}_{\mathrm{H}}=4 \pi|k| \gamma_{k}
$$

which, being one of Engman's main results, we have shown it can be applied to the CCLP BH.

\section{Discussion and Final Conclusions}

At this point, a couple of comments are in order: (i) We emphasize that, due to the writing we have performed, Engman's results can be trivially applied to Equation (6); (ii) The expression for the area of the event horizon in Equation (24) can be considered a generic result for certain metrics. Specifically, it is well known [28] that an $S^{1}$ invariant two dimensional manifold diffeomorphic to the sphere with mirror symmetry about its equator is uniquely determined by its spectrum. This has been generalized by Zelditch [29] for certain surfaces of revolution. Interestingly, the class of metrics considered by Zelditch include the Kerr-Newman [11] and the CCLP event horizons as we have shown here. Even more, whether this property may hold more generally for certain classes of $\mathrm{BH}$ event horizons can be tested by looking at Zelditch's requirements [29]:

1. $S^{1}$ invariance implies the existence of two fixed points (let us call north $(\mathrm{N})$ and south (S) poles).

2. In geodesic polar coordinates centered at $\mathrm{N}$, the metric can be written as $g=d r^{2}+$ $a(r)^{2} d \theta^{2}$. 
3. $a(r)$ is real analytic.

4. $a(r)$ has one critical point at $r_{0}$ with $a^{\prime \prime}\left(r_{0}\right)<0$. This critical point corresponds to a "equatorial geodesic".

5. The Poincare map for the equatorial geodesic is of twist type.

Up to our knowledge, conditions 1-3 are usually satisfied for all BH event horizon surfaces. However, condition 3 rules out planar and hyperbolic horizons. Finally, condition 5 is included to ensure that there are global action-angle variables for the geodesic flow and it should be evaluated in any particular case of interest.

Finally, we present a brief summary of the main findings shown by Engman regarding the Kerr-Newman [11] and the de Sitter-Kerr-Newman [13] solutions together with the case here studied.

\subsection{Kerr-Newman}

In this case, the metric of the Kerr-Newman event horizon can be determined with $\gamma_{0}$ and $\gamma_{1}$. One can reconstruct this metric by using $\gamma_{0}$ together with $\gamma_{k}$ for any $k$. In addition, all the physical parameters $(m, a, Q)$ can also be written in terms of the spectra. Even more, by using Robinson's uniqueness theorem [12], Engman shows that the whole spacetime (for the uncharged case) can be explicitly constructed from the spectra of the Laplacian on the event horizon.

\subsection{Kerr-Newman-de Sitter}

When a positive cosmological constant is added, Engman shows [13] that all the parameters of the solution $(m, a, Q, \Lambda)$ can be given in terms of two different types of trace formulae associated with the Laplacian on the event and the cosmological horizon.

\subsection{Chong-Cvetic-Lu-Pope}

In this case, the constraint $\Delta_{r}=0$ give four horizons as in the de Sitter-Kerr-Newman solution. Unfortunately, due to the intricate form of $\Delta_{r}$ (due to the coupling between the charges and the intensity of the scalar potential) compared with that of the de Sitter-KerrNewman case, we were not able to express the parameters of the metric in terms of the eigenvalues of the Laplacian. However, we were able to adapt the strategy follow by Smarr to write the area of the event horizon in a convenient way and as a consequence, we demonstrated that it can be constructed in terms of the spectra of the Laplacian, as in the (de Sitter-)Kerr-Newman cases.

Regarding the physical interpretation of the Laplace spectra of the event horizon, several comments are in order. As pointed out by Engman [11], (i) the quantities $\gamma_{k}$ are fundamental quantities coming from the discrete set of vibrational wave frequencies on the event horizon (ii) the angular momentum operator coming from the separation of variables on Teukolsky's Equation [30] is closely related to the Laplacian (iii) quasinormal mode frequencies are also related to the separation constants in the former separation of variables and (iv), from the holographic point of view, the importance of the spectra, which determines the area of the event horizon, is guaranteed. In the case here studied, the determination of the area from the spectra can serve as a preliminary step towards an understanding of the string (or other supergravity theories) degrees of freedom in terms of the spectra of certain canonical operators defined on the event horizon. We hope that other authors will pursue this line of research in order to see the robustness of this idea.

Author Contributions: Conceptualization, P.B.; methodology, P.B.; writing-original draft preparation, P.B., E.C. and J.M.P.; writing-review and editing, P.B., E.C. and J.M.P. All authors have read and agreed to the published version of the manuscript.

Funding: P.B. is funded by the Beatriz Galindo contract BEAGAL 18/00207 (Spain). J.M.P. is funded by the projects ANT1956 and SEM18-02 of the University of Antofagasta (Chile).

Acknowledgments: P.B. acknowledges Anaís, Lucía, Inés and Ana for continuous support. 
Conflicts of Interest: The authors declare no conflict of interest.

\section{References}

1. Weyl, H. Über die asymptotische Verteilung der Eigenwerte; Nachr. Königl. Ges. Wiss.: Göttingen, Germany, $1911 ;$ Volume 1, p. 110.

2. Berger, M.; Gauduchon, P.; Mazet, E. Le Spectre d'une Variété Riemannienne, Lecture Notes in Mathematics; Springer: Berlin, Germany; New York, NY, USA, 1971; Volume 194.

3. Chamseddine, A.H.; Connes, A. Universal Formula for Noncommutative Geometry Actions: Unification of Gravity and the Standard Model. Phys. Rev. Lett. 1996, 77, 4868. [CrossRef]

4. Chamseddine, A.H.; Connes, A. The Spectral Action Principle. Commun. Math. Phys. 1997, 186, 731. [CrossRef]

5. Landi, G.; Rovelli, C. General Relativity in Terms of Dirac Eigenvalues. Phys. Rev. Lett. 1997, 78, 305. [CrossRef]

6. Aasen, D.; Bhamre, T.; Kempf, A. Shape from Sound: Toward New Tools for Quantum Gravity. Phys. Rev. Lett. 2013, 110, 121301. [CrossRef] [PubMed]

7. Panine, M.; Kempf, A. Towards spectral geometric methods for Euclidean quantum gravity. Phys. Rev. D 2016, $93,084033$. [CrossRef]

8. Yazdi, Y.K.; Letizia, M.; Kempf, A. Lorentzian spectral geometry with causal sets, Class. Quantum Grav. 2021, $38,015011$. [CrossRef]

9. Datchev, K.; Hezari, H. Inverse Problems and Applications: Inside Out II; Math. Sci. Res. Inst. Publ. Series, 60, Section 10; Cambridge University Press: Cambridge, UK, 2012.

10. Panine, M.; Kempf, A. A convexity result in the spectral geometry of conformally equivalent metrics on surfaces. Int. J. Geom. Meth. Mod. Phys. 2017, 14, 1750157. [CrossRef]

11. Engman, M.; Cordero-Soto, R. Intrinsic spectral geometry of the Kerr-Newman event horizon. J. Math. Phys. 2006, 47, 033503. [CrossRef]

12. Robinson, D.C. Uniqueness of the Kerr Black Hole. Phys. Rev. Lett. 1975, 34, 905. [CrossRef]

13. Engman, M.; Santana, G.A. Spectral geometry of cosmological and event horizons for Kerr-Newman-de Sitter metrics. Proc. Am. Math. Soc. 2013, 141, 3305. [CrossRef]

14. Chong, Z.W.; Cvetic, M.; Lu, H.; Pope, C.N. Charged Rotating Black Holes in Four-Dimensional Gauged and Ungauged Supergravities. Nucl. Phys. B 2005, 717, 246. [CrossRef]

15. Cvetic, M.; Gibbons, G.W.; Lu, H.; Pope, C.N. Rotating Black Holes in Gauged Supergravities; Thermodynamics, Supersymmetric Limits, Topological Solitons and Time Machines. arXiv 2005, arXiv:hep-th/0504080.

16. Daniel, Z. Freedman and Antoine Van Proeyen, Supergravity; Cambridge University Press: Cambridge, UK, 2012.

17. Stelle, K. Lectures on Supergravity p-branes. arXiv 1997, arXiv:hep-th/9701088.

18. Becker, K.; Becker, M.; Schwarz, J.H. String Theory and M-Theory: A Modern Introduction; Cambridge University Press: Cambridge, $\mathrm{UK}, 2007$.

19. Samtleben, H. Lectures on Gauged Supergravity and Flux Compactifications. Class. Quant. Grav. 2008, 25, 214002. [CrossRef]

20. Ortin, T. Gravity and Strings, Cambridge Monographs on Mathematical Physics, 2nd ed.; Cambridge University Press: Cambridge, UK, 2015.

21. Peet, A. TASI lectures on black hole in strings theory. In Strings, Branes and Gravity; TASI 99; Harvey, J.A., Kachru, S., Silverstein, E., Eds.; Worldscientific: Hackensack, NJ, USA, 2001.

22. Cvetic, M.; Lu, H.; Pope, C.N. Four-dimensional $N=4$, SO(4) gauged supergravity from $D=11$. Nucl. Phys. B 2000, 574, 761. [CrossRef]

23. Duff, M.J.; Stelle, K.S. Multi-membrane solutions of d = 11 supergravity. Phys. Lett. B 1991, 253, 113-118. [CrossRef]

24. Smarr, L. Surface Geometry of Charged Rotating Black Holes. Phys. Rev. D 1973, 7, 289. [CrossRef]

25. Engman, M. New spectral characterization theorems for $S^{2}$. Pac. J. Math. 1992, 154, 215. [CrossRef]

26. Engman, M. Trace formulae for $S^{1}$ invariant Green's operators on $S^{2}$. Manuscripta Math. 1997, 93, 357. [CrossRef]

27. Engman, M. Sharp bounds for eigenvalues and multiplicitites on surfaces of revolution. Pac. J. Math. 1998, 186, 29-37. [CrossRef]

28. Bruning, J.; Heintze, E. Spektrale Starrheit gewisser Drehflächen. Math. Ann. 1984, 269, 95-101. [CrossRef]

29. Zelditch. S. The inverse spectral problem for surfaces of revolution. J. Differ. Geom. 1998, 49, 207-264. [CrossRef]

30. Teukolsky, S.A. Rotating black holes-Separable wave equations for gravitational and electromagnetic perturbations. Phys. Rev. Lett. 1972, 29, 1114. [CrossRef] 